\title{
Prospects for Measuring the Hubble Constant with Neutron-Star-Black-Hole Mergers
}

\author{
Stephen M. Feeney $\odot,{ }^{1,{ }^{*}}$ Hiranya V. Peiris $\odot,{ }^{1,2}$ Samaya M. Nissanke, ${ }^{3,4}$ and Daniel J. Mortlock ${ }^{5,6,7}$ \\ ${ }^{1}$ Department of Physics \& Astronomy, University College London, Gower Street, London WC1E 6BT, United Kingdom \\ ${ }^{2}$ Oskar Klein Centre for Cosmoparticle Physics, Department of Physics, Stockholm University, \\ AlbaNova, Stockholm SE-106 91, Sweden \\ ${ }^{3}$ GRAPPA, Anton Pannekoek Institute for Astronomy and Institute of High-Energy Physics, University of Amsterdam, \\ Science Park 904, 1098 XH Amsterdam, Netherlands \\ ${ }^{4}$ Nikhef, Science Park 105, 1098 XG Amsterdam, Netherlands \\ ${ }^{5}$ Astrophysics Group, Imperial College London, Blackett Laboratory, Prince Consort Road, London SW7 2AZ, United Kingdom \\ ${ }^{6}$ Department of Mathematics, Imperial College London, London SW7 2AZ, United Kingdom \\ ${ }^{7}$ Department of Astronomy, Stockholm University, AlbaNova, SE-10691 Stockholm, Sweden
}

(Received 19 January 2021; revised 9 March 2021; accepted 12 March 2021; published 28 April 2021)

Gravitational wave (GW) and electromagnetic (EM) observations of neutron-star-black-hole (NSBH) mergers can provide precise local measurements of the Hubble constant $\left(H_{0}\right)$, ideal for resolving the current $H_{0}$ tension. We perform end-to-end analyses of realistic populations of simulated NSBHs, incorporating both GW and EM selection for the first time. We show that NSBHs could achieve unbiased $1.5 \%-2.4 \%$ precision $H_{0}$ estimates by 2030 . The achievable precision is strongly affected by the details of spin precession and tidal disruption, highlighting the need for improved modeling of NSBH mergers.

DOI: 10.1103/PhysRevLett.126.171102

Introduction.-The current expansion rate of the Universe - the Hubble constant $H_{0}$-is at the heart of a significant cosmological controversy. Direct measurements in the local Universe by the SHOES team's Cepheid-supernova distance ladder [1] find $H_{0}=$ $74.03 \pm 1.42 \mathrm{~km} \mathrm{~s}^{-1} \mathrm{Mpc}^{-1}$. This is discrepant at the $4.4-\sigma$ level from the $67.36 \pm 0.54 \mathrm{~km} \mathrm{~s}^{-1} \mathrm{Mpc}^{-1}$ value inferred from the Planck satellite's observations of the cosmic microwave background (CMB) anisotropies, assuming the standard flat cosmological model [2].

There are two potential explanations for this discrepancy, the most exciting of which derives from the modeldependence of the CMB constraint: could the discrepancy be due to physics beyond the standard model? Despite extensive effort (e.g., Refs. [3,4]), consensus on a compelling theoretical explanation has not been reached. The more prosaic explanation posits undiagnosed systematic errors or underestimated uncertainties; however, despite multiple investigations of both the distance ladder [5] and CMB [6] datasets, no study has found incontrovertible evidence warranting a change of conclusions.

In the absence of conclusive evidence of systematic errors or consensus on an extended model, independent verifications of the two central measurements offer a promising route to resolving the tension. Independent verification of the CMB anisotropy constraints comes from recent inverse distance ladder datasets [7]. Local verification has, however, proven more challenging, with some alternative analyses supporting the SHOES team's findings [8] and others providing contradictory conclusions of varying significance [9], in some cases using the same data.

A direct, completely independent local measurement with percent-level precision is therefore needed to resolve the $H_{0}$ tension. Combined gravitational-wave (GW) and electromagnetic (EM) observations of nearby compactobject mergers are ideal candidates to provide that measurement, yielding $H_{0}$ estimates that depend on general relativity alone [10-33]. Thanks to their accompanying EM emission, the utility of binary neutron star (BNS) mergers is well established [12-25], but less attention has been paid to the potential contribution of as-yet undiscovered neutronstar-black-hole (NSBH) mergers with EM counterparts $[13,16,34]$. Using idealized, fixed-signal-to-noise simulations at indicative parameter values, Vitale and Chen [34] recently showed that catalogs of GW-selected NSBH observations may constrain $H_{0}$ as well as BNSs, depending on the relative merger rates and $\mathrm{BH}$ spins. In particular, they showed explicitly that luminosity distance estimates could improve, as misaligned $\mathrm{BH}$ spins induce spin precession, helping break the degeneracy between the luminosity distance and inclination angle for some NSBH systems.

Here, we determine the $H_{0}$ constraints realistic NSBH samples will achieve, by performing end-to-end analyses of simulated NSBH samples incorporating fully specified parent populations, combined GW and EM selection, and a complete noise treatment. We use state of the art GW waveforms [35,36] and EM outflow models [37], both calibrated to a suite of numerical relativity simulations, for 
our GW and EM signals, and focus on the "A+" era of the mid-to-late 2020s, assuming an expanded GW network including LIGO India and KAGRA.

Simulations. - In this work we simulate the results of a circa-2025 GW detector network, consisting of LIGO A+, Virgo AdV+, KAGRA, and LIGO India [38,39] observing for $t_{\mathrm{obs}}=5 \mathrm{yr}$ with duty cycle of $\Delta_{\text {obs }}=0.5$. We assume a constant rest-frame NSBH merger rate $\Gamma_{\text {fid }}=610 \mathrm{yr}^{-1} \mathrm{Gpc}^{-3}$ (corresponding to the 90\% upper limit of Ref. [40]), and cosmological parameters matching Ref. [2], with $H_{0}=67.36 \mathrm{~km} \mathrm{~s}^{-1} \mathrm{Mpc}^{-1}$ and $q_{0}=-0.527$. Each simulation proceeds by drawing the total number of mergers from a Poisson distribution with mean $\lambda=\Delta_{\text {obs }} t_{\mathrm{obs}} V \Gamma_{\text {fid }}$, where $V$ is the redshifted volume calculated using a third-order cosmographic comoving volume element (see Supplemental Material [41] for details). To reduce computation time, the volume integral can be truncated at some redshift $z_{\max }$ where there is negligible probability of even the loudest merger being detected: we find that $z_{\max }=0.44$ suffices for our setting. For our fiducial parameter set, the mean number of mergers $\lambda=25160$; our particular Poisson draw yields a preselection total of 25241 .

For each merger, we draw a cosmological redshift $z$, assuming a constant source-frame rate [see, e.g., Eq. (28) of Ref. [24] ], along with an isotropically distributed angular sky position, inclination angle phase, and polarization angle. We draw uniformly distributed $\mathrm{BH}$ masses from $P\left(m_{\mathrm{BH}}\right)=\mathrm{U}\left(2.5 M_{\odot}, 40 M_{\odot}\right)$, taking the upper limit from low-metallicity binary population synthesis simulations [42] (using solar metallicities would reduce this upper limit to $12 M_{\odot}$ ) and extending to low masses to reflect the detection of objects in the purported NS/BH mass gap [43]. NS masses are drawn from $P\left(m_{\mathrm{NS}}\right)=\mathrm{U}\left(1 M_{\odot}, 2.42 M_{\odot}\right)$, with an upper limit chosen to match that of the DD2 equation of state (EOS) [44]. Dimensionless BH and NS spin magnitudes are drawn from the uniform distributions $P\left(a_{\mathrm{BH}}\right)=\mathrm{U}(0,0.99) \quad$ and $P\left(a_{\mathrm{NS}}\right)=\mathrm{U}(0,0.05)$ and are assumed to be isotropically oriented. Following Refs. [37,45], we use the component masses, NS compactness, and BH spins to calculate the baryonic mass ejected by each merger. This formula requires the assumption of a NS EOS (we again use DD2) and has been calibrated using simulations without precession due to misaligned $\mathrm{BH}$ spins. We use the same EOS to calculate tidal deformabilities for the NSs, and set the BH deformabilities to zero [46]. Finally, we generate a peculiar velocity $v$ for each merger from a zero-mean normal with a standard deviation of $500 \mathrm{~km} \mathrm{~s}^{-1}$.

With the NSBH parameters in hand, we generate mock data for each merger and apply our selection criteria. To determine the impact of different physical effects on our results, we simulate two populations using different waveform approximants: the BNS-calibrated IMRPhenomPv2_NRTidal [35] and NSBH-specific
SEOBNRV4_ROM_NRTidalv2_NSBH [36] (hereafter IMRPhenom and SEOBNR). We refer the reader to the Supplemental Material [41] (which includes Refs. [47-49]) for a complete discussion of the differences between the two waveforms. The SEOBNR waveform requires aligned or antialigned spins, so we set the transverse NS and BH spins to zero after sampling them isotropically (mimicking, in some sense, spins becoming aligned over time). For each merger, we generate a 32-sec segment of noisy (using spectra from Ref. [39]) GW data $\hat{\boldsymbol{x}}$ per waveform using the same random seed and a frequency range of $20-2048 \mathrm{~Hz}$, considering it detected if the network signal-to-noise ratio (SNR) is at least $\rho_{*}=12$. We assume that the GW detectors operate in concert with an EM follow-up program capable of detecting all mergers with ejecta mass greater than $m_{\mathrm{ej}}^{*}=0.01 M_{\odot}$, modeling for the first time a hybrid GWEM selection function for NSBHs. This ejecta material is assumed to produce EM emission in the form of a gammaray burst, kilonova and/or afterglow, as opposed to the "battery" effect of Ref. [50]. Finally, we generate noisy measured redshifts and peculiar velocities by drawing from $P(\hat{z} \mid z)=N(z, 0.001)$ and $P(\hat{v} \mid v)=N\left(v, 200 \mathrm{~km} \mathrm{~s}^{-1}\right)$, respectively. Of the 25241 simulated mergers, 2477 (2954) are detected in GWs using the IMRPhenom (SEOBNR) waveform, 99 (75) of which have sufficient ejecta to be detected in EM; 62 appear in both samples. The SNRs for SEOBNR waveforms are, on average, $5.9 \%$ larger than their IMRPhenom counterparts, resulting in the GW-detected SEOBNR sample containing 500 more objects. (We hypothesise that this is due to the effects of generic spin precession on the IMRPhenom population. Differences in the lengths of GW signals within the detector frequency bands due to the two waveforms' different merger frequencies would tend to boost the IMRPhenom SNRs.) Setting the transverse spins to zero for use with the SEOBNR waveform, however, has the side effect of reducing the typical ejecta mass [37] and hence the final GW + EM-detected sample.

The impact of our selection function is illustrated in Fig. 1, in which we plot histograms of our full population (dotted lines), GW-selected events (dashed lines), and GW + EM-selected mergers (colored bars) for a subset of our parameters. The prior curves are identical in both cases apart from the BH spin magnitudes, where zeroing the transverse spins has made the SEOBNR population's distribution nonuniform. The primary impact of the GW SNR threshold is, as expected, to select nearby mergers; it also imparts very slight preferences for low mass ratios and prograde $\mathrm{BH} z$ spins [51]. It is interesting to note that the GW-selected SEOBNR distance distribution is broader than that of IMRPhenom and peaked at slightly higher distances: this is a direct consequence of the SEOBNR injections' systematically higher SNRs. Further, the presence of spin precession permits the detection of more edge-on IMRPhenom waveforms. 


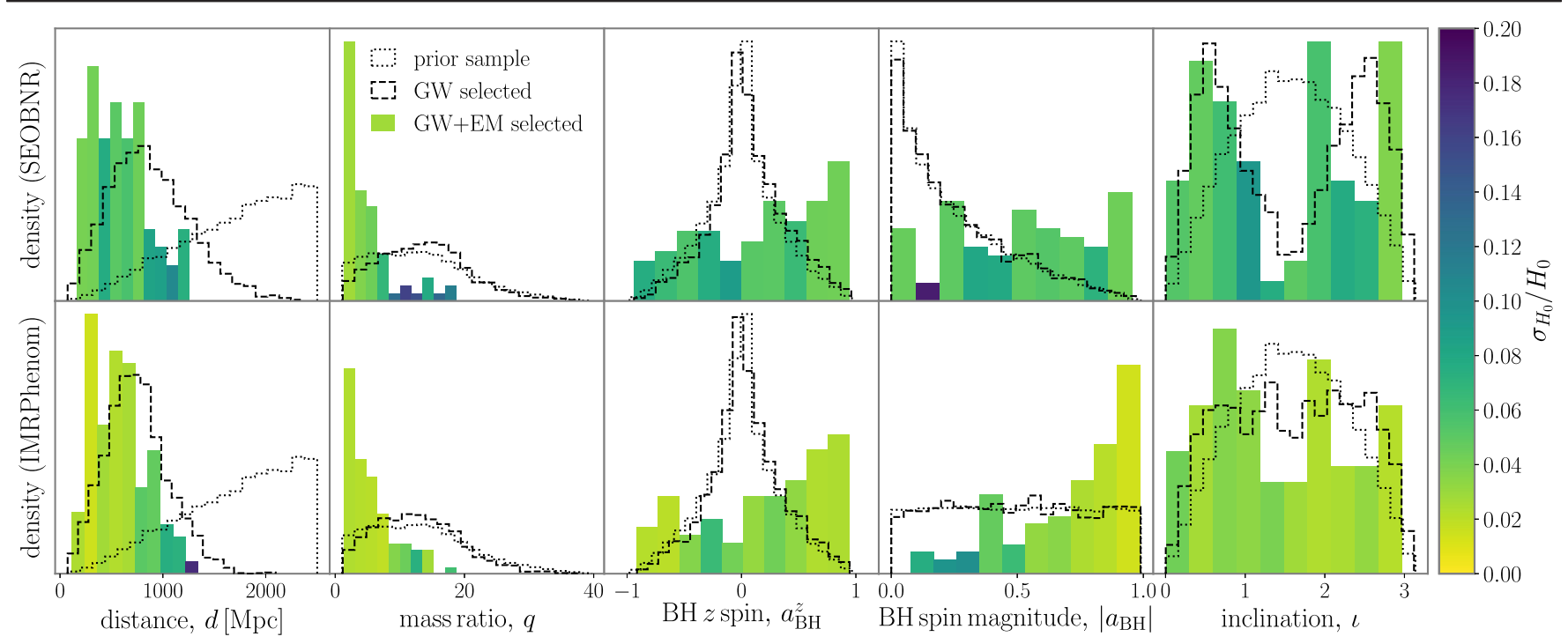

FIG. 1. Distributions of a subset of parameters from our SEOBNR (top) and IMRPhenom (bottom) samples, as drawn from the prior (dotted), selected by GW SNR (dashed) and selected by GW and EM emission (colored histograms). The bins are colored by the fractional $H_{0}$ uncertainty the mergers within the bin achieve: the yellowest and lightest bins are most informative.

The ejecta-mass threshold (i.e., EM selection) strongly impacts the observed distributions. The GW + EM-detected distributions are shifted to even smaller distances, particularly for the SEOBNR waveform, as the low-mass-ratio systems which produce significant ejecta mass can only be detected nearby. There is a very strong preference for mass ratios under 10 (again, especially so for SEOBNR) and large spins [37,45], and the preference for positive $z$ spins is much more pronounced. (Populations with $\sim$ solar metallicities produce NSBHs with lower $\mathrm{BH}$ masses [42] and hence more GW + EM-detectable mergers.) As expected from Refs. $[37,45,50]$, the bulk of detected systems have BH masses below $10 M_{\odot}$. The differences between the two waveforms' GW + EM-selected distributions are slightly obfuscated by the small sample sizes, but the SEOBNR sample is shifted towards lower distances and mass ratios. As the SEOBNR mergers' BH spin magnitudes are smaller than those of their IMRPhenom counterparts, they require smaller mass ratios to produce significant ejecta [37], and the resulting systems are harder to detect at distance. Our implementation of EM selection captures the current best understanding of the dependence on ejecta mass, but we note that a fully self-consistent model of EM selection does not yet exist. This selection does not, for example, incorporate any viewing-angle dependence (see Ref. [52] for a treatment in BNS mergers) or EM survey selection effects [53-56].

Methods.-The probabilistic inference of the Hubble constant from catalogs of compact object mergers has been described in detail in the literature [10,12-14,16,19, $21-25,57,58]$. In the following, we adopt a slight variant of the formalism set out in Ref. [24], whose Fig. 9 depicts a network diagram for the model we use to describe the data. (The only addition required to the network diagram of
Ref. [24] is the dependence of the selection $S$ on an intrinsic parameter: the merger's ejecta mass.) The precise posterior we evaluate is defined in the Supplemental Material [41].

We infer the parameters of this model in two parts, using two sampling methods. First, we process each merger individually in order to obtain the GW likelihoods marginalized over all parameters $\boldsymbol{\theta}_{i}$ other than the luminosity distance $d$ to the merger $\left(_{i}\right.$ here comprising the $i$ th merger's component masses, spin magnitudes and orientations where used, inclination, polarization angle, NS tidal deformability, and time and phase at coalescence). We adopt priors identical to the distributions used in the generative model for all parameters other than the masses. Convergence is greatly improved by sampling chirp masses and mass ratios instead of component masses, and we therefore sample using interim priors that are uniform in these parameters (over the ranges permitted by our component-mass extrema), before importance sampling the outputs to reinstate our desired component-mass priors. The marginal GW likelihoods are sampled with the pypolychord nested sampler [59], wrapped by bilby [60], using 1000 live points and bilby's marginalize_phase, _time and_distance settings. Each 15 (11)-dimensional IMRPhenom (SEOBNR) sampling run takes 6-14 (4-6) days to complete on one Intel Xeon $2.7 \mathrm{GHz}$ CPU.

Given the marginal GW likelihoods, we use No-U-Turn Sampling as implemented by the pystan package [61] to infer the cosmological and population parameters. To connect to the cosmological parameters, we adopt a third-order distance-redshift relation matching our volume element [62], with $H_{0}$ and $q_{0}$ allowed to vary but the jerk set to one. We assume a broad Gaussian prior on $H_{0}$, 
$P\left(H_{0}\right)=N(70,20) \mathrm{km} \mathrm{s}^{-1} \mathrm{Mpc}^{-1}$, a truncated Gaussian prior on $q_{0}, P\left(q_{0}\right)=\Theta\left(q_{0}+2\right) \Theta\left(1-q_{0}\right) N(-0.5,0.5)$, and a $\log$-uniform prior on the rate, $P(\Gamma) \propto 1 / \Gamma$. To use pystan, we must be able to sample all parameters from analytic distributions. We therefore perform a Gaussian mixture model fit to each merger's marginal distance likelihood using pomegranate [63]. We fit each likelihood with an integer grid of 2-10 mixture components, repeating 10 times at each grid point and selecting the best fit using the Akaike information criterion [64].

Finally, we must evaluate the expected number of detected mergers $\bar{N}$ at each sampled value of the cosmological and population parameters. We do so by resimulating the catalogs 100 times at each point of a $5 \times 5$ grid in $\left\{H_{0}, q_{0}\right\}$ assuming our fiducial rate, $\Gamma_{\text {fid }}$, and interpolating the results using a $2 \mathrm{D}$ fourth-order interpolation. The dependence on the sampled rate is captured by multiplying the interpolation coefficients by $\Gamma / \Gamma_{\text {fid }}$. The resulting 153 (193)-dimensional pystan inference runs take less than a minute to generate 20000 well converged samples on a $3.1 \mathrm{GHz}$ Intel Core i7 CPU. The set of true redshifts and peculiar velocities are uninteresting for the purposes of cosmology inference, and we marginalize over these parameters when quoting the results below.

Results.-Processing the simulated SEOBNR and IMRPhenom catalogs through our two-stage inference pipeline produces the cosmology and population parameter posteriors shown in the Supplemental Material [41]. In both cases, the recovered $H_{0}, q_{0}$, and rate posteriors are completely consistent with the input values, indicating, as expected, that the selection effects are correctly accounted for [24]. The $68 \%$ credible intervals on the near-Gaussian $H_{0}$ marginal posteriors are $68.8 \pm$ $1.6 \mathrm{~km} \mathrm{~s}^{-1} \mathrm{Mpc}^{-1}$ for the SEOBNR sample and $66.5 \pm$ $1.0 \mathrm{~km} \mathrm{~s}^{-1} \mathrm{Mpc}^{-1}$ for IMRPhenom. As the IMRPhenom sample contains 99 objects to the SEOBNR sample's 75, we should therefore expect that the IMRPhenom sample's $H_{0}$ posterior be roughly $13 \%$ narrower than that of the SEOBNR sample. The remaining reduction, therefore, reflects the ability for precessing spins to break the distance-inclination degeneracy [21,65-67]. This additional constraining power is equivalent to an approximate doubling of the catalog size.

The $H_{0}$ uncertainties we find for both waveforms are comparable to the current Cepheid-SN distance ladder precision [1]. NSBH populations-should they produce EM counterparts and occur at rates roughly matching our assumptions-will therefore strongly inform the outcome of the current $H_{0}$ tension, particularly when combined with accompanying BNS populations, likely of comparable size [21,23,24,34]. (We note that comparable $H_{0}$ precision had been hoped for by 2023 from catalogs of BNS mergers [21]. That timescale, however, now appears optimistic due to the lack of EM counterpart detections following GW170817.) The mergers are also informative about the deceleration parameter, $q_{0}$, shrinking its uncertainty from 0.5 to 0.32 or 0.27 , depending on the waveform. This further implies that NSBH catalogs will be able to begin constraining parameters such as the matter density and dark energy EOS (in the context of $\Lambda \mathrm{CDM}$ and extended models), complementary to BBH results from higher redshifts $[31,33,68]$. The merger rates are recovered with roughly $10 \%$ precision $[40,43,69,70]$.

To obtain a picture of the parameter combinations that are most important for the $H_{0}$ constraints, we return to Fig. 1. Here, the colors of the histogram bins indicate the fractional $H_{0}$ uncertainty the mergers within each bin attain, with the most-constraining bins colored yellow and the least-constraining blue. For both waveforms, the bulk of the $H_{0}$ constraining power comes from mergers out to roughly $700 \mathrm{Mpc}$, not just the very nearest ( 200 Mpc), loudest events. For the IMRPhenom mergers, all mass ratio bins less than $\sim 8 M_{\odot}$ contribute equally, despite the frequency dropping rapidly with mass ratio. For the SEOBNR mergers, the constraints are instead driven by the lowest-mass ratio events [65,66]. From the IMRPhenom spin panels, it is clear that highest-spin events constrain $H_{0}$ most strongly, with the full $H_{0}$ constraint coming almost entirely from the highest-spin (and most populated) bin. The SEOBNR constraint, on the other hand, is sourced by events with a broader range of spins, though this is likely driven by the prior.

We further highlight the importance of precession in breaking the distance-inclination degeneracy in Fig. 2. In the first three panels we plot distance and inclination constraints for a selection of mergers when using the SEOBNR (red, filled) and IMRPhenom (grey, filled) waveforms. For the first two mergers, detected with high SNR, the long degeneracies present in their SEOBNR posteriors are almost completely broken when using the IMRPhenom waveform, in which the spins precess. We illustrate this point further by re-running the IMRPhenom case assuming aligned spins, having set the transverse spins to zero. These results are overlaid as dashed dark red contours. Both distance-inclination degeneracies blow up, increasing the distance uncertainties by a factor of over three, with commensurate consequences for the mergers' ability to constrain $H_{0}$. In the third panel, we show equivalent posteriors for the IMRPhenom merger whose $\mathrm{BH}$ spin is closest to being aligned: the effect of switching waveforms here is markedly reduced. The impact on the population level is clear from the right-hand panel of Fig. 2, in which we plot the distributions of individual mergers' fractional errors on distance (dashed) and $H_{0}$ (solid) when using the SEOBNR (red) and IMRPhenom (gray) waveforms. The SEOBNR distributions are shifted to significantly higher errors than their IMRPhenom counterparts, despite the SEOBNR mergers typically having higher SNRs. The smallest percentage error for any individual merger is $2.8 \%$ for the IMRPhenom case and $6.1 \%$ for 


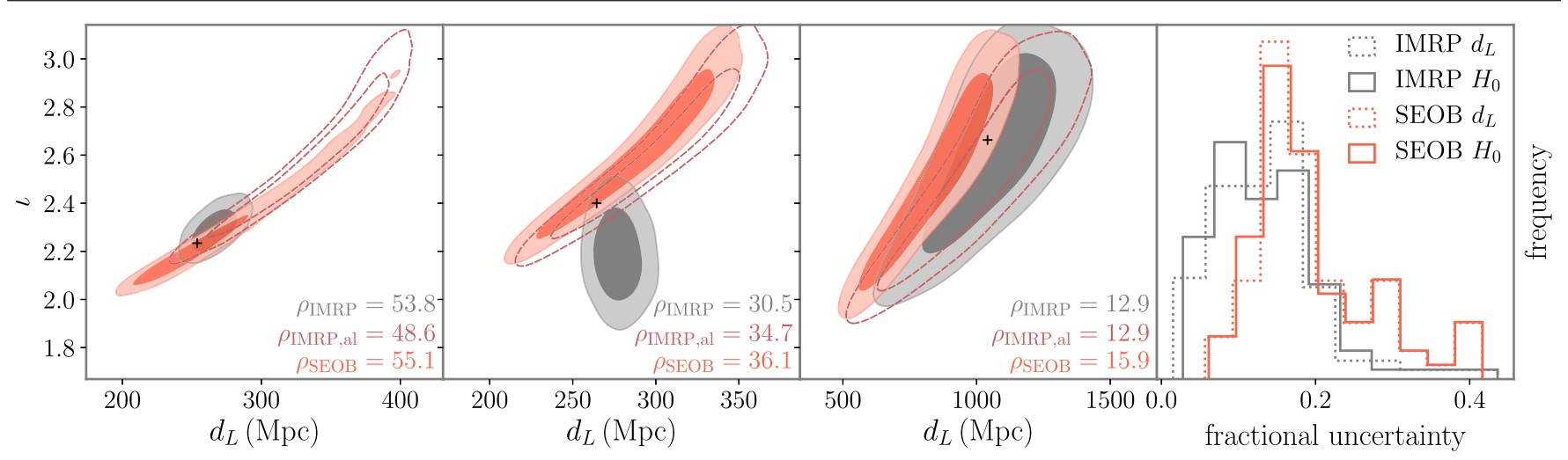

FIG. 2. Left three panels: distance and inclination posteriors for a selection of mergers, simulated and sampled using the IMRPhenom waveform with precessing (gray filled) and aligned (dark red dashed) spins, and using SEOBNR with aligned spins (red). The selection includes the highest-SNR merger common to both catalogs (left) and the IMRPhenom merger whose BH spin is closest to being aligned (second from right). Right: distributions of fractional uncertainties on luminosity distance (dotted) and $H_{0}$ (solid) from individual mergers from our IMRPhenom (gray) and SEOBNR (red) NSBH catalogs.

SEOBNR; the medians are $13.2 \%$ and $17.3 \%$, respectively. The $H_{0}$ constraints imparted by both "golden" and normal events are therefore stronger when spins precess significantly. Finally, we note from the lower limits of the dashed curves that peculiar velocity and redshift uncertainties strongly suppress the constraining power of the nearest and loudest events.

Conclusions. - In this Letter, we present the results of the first end-to-end inference of $H_{0}$ from realistic simulated catalogs of NSBH mergers incorporating GW and EM selection effects. The precision we should expect from such catalogs is very promising for resolving the current $H_{0}$ tension, with five years of $\mathrm{A}+$ era observations yielding $H_{0}$ uncertainties of $1.5 \%-2.4 \%$. We find, however, that the detailed physics of the NSBH waveforms strongly impacts the achievable precision. Using the SEOBNRV4 ROM NRTidalv2 NSBH waveform with nonprecessing $\mathrm{BH}$ spins results in boosted SNRs, and an increase of $\sim 500 \mathrm{GW}$-detected NSBHs. However, including precessing spins using the IMRPhenomPv2_NRTidal waveform markedly increases the typical ejecta mass and hence the number of combined GW + EM detections. Critically, precessing spins also break the distanceinclination degeneracy in the resulting GW parameter posteriors, yielding a significant improvement ( $\sim 40 \%$ after accounting for differing catalog sizes) in the resulting $H_{0}$ constraint. Our results strongly highlight the need for improved modeling of NSBH signals in both gravitational waves (see, e.g., Ref. [71]) and the electromagnetic spectrum.

The Python simulation and inference software developed for this analysis are publicly available [72].

We thank Sukanta Bose for providing details on LIGO India, Nikhil Sarin and Greg Ashton for help with bilby, Will Handley for help with pypolychord, and Tanja Hinderer, Andrew Williamson, Francois Foucart, and
Bastien DuBoeuf for useful discussions. S. M. F. is supported by the Royal Society. H. V. P.'s work was partially supported by the research environment grant "Gravitational Radiation and Electromagnetic Astrophysical Transients (GREAT)" funded by the Swedish Research Council (VR) under Dnr 2016-06012 and the research project grant "Gravity Meets Light" funded by the Knut and Alice Wallenberg Foundation Dnr KAW 2019.0112. S. M. N. is grateful for financial support from the Nederlandse Organisatie voor Wetenschappelijk Onderzoek (NWO) through the VIDI and Projectruimte grants. H. V.P. and D. J. M. acknowledge the hospitality of the Aspen Center for Physics, which is supported by National Science Foundation Grant No. PHY-1607611. The participation of H. V. P. and D. J. M. at the Aspen Center for Physics was supported by the Simons Foundation. This work used computing facilities provided by the UCL Cosmoparticle Initiative; and we thank the HPC systems manager Edd Edmondson for his dedicated support. We are deeply grateful to the IMRPhenom and SEOBNR waveform modelers for making these waveforms public, without which this work would not have been possible. S. M.F. participated in conceptualization, methodology, software, investigation, validation, and writing (original draft preparation). H. V. P. participated in conceptualization, methodology, validation, writing (review and editing), and funding acquisition. S. M.N. participated in conceptualization, methodology, validation, and writing (review and editing). D. J. M. participated in conceptualization, methodology, and writing (review and editing).

*stephen.feeney@ucl.ac.uk

[1] A. G. Riess, S. Casertano, W. Yuan, L. M. Macri, and D. Scolnic, Large magellanic cloud cepheid standards provide a $1 \%$ foundation for the determination of the hubble 
constant and stronger evidence for physics beyond $\Lambda \mathrm{CDM}$, Astrophys. J. 876, 85 (2019).

[2] Planck Collaboration, Planck 2018 results. VI. Cosmological parameters, Astron. Astrophys. 641, A6 (2020).

[3] L. Knox and M. Millea, Hubble constant hunter's guide, Phys. Rev. D 101, 043533 (2020).

[4] S. Vagnozzi, New physics in light of the $\mathrm{H}_{0}$ tension: An alternative view, Phys. Rev. D 102, 023518 (2020).

[5] G. Efstathiou, $\mathrm{H}_{0}$ revisited, Mon. Not. R. Astron. Soc. 440, 1138 (2014); M. Rigault et al., Confirmation of a star formation bias in Type Ia supernova distances and its effect on the measurement of the Hubble constant, Astrophys. J. 802, 20 (2015); D. O. Jones, A. G. Riess, and D. M. Scolnic, Reconsidering the effects of local star formation on Type Ia supernova cosmology, Astrophys. J. 812, 31 (2015); W. Cardona, M. Kunz, and V. Pettorino, Determining $\mathrm{H}_{0}$ with Bayesian hyper-parameters, J. Cosmol. Astropart. Phys. 03 (2017) 056; B. R. Zhang, M. J. Childress, T. M. Davis, N. V. Karpenka, C. Lidman, B. P. Schmidt, and M. Smith, A blinded determination of $\mathrm{H}_{0}$ from low-redshift Type Ia supernovae, calibrated by Cepheid variables, Mon. Not. R. Astron. Soc. 471, 2254 (2017); B. Follin and L. Knox, Insensitivity of the distance ladder Hubble constant determination to Cepheid calibration modelling choices, Mon. Not. R. Astron. Soc. 477, 4534 (2018); S. M. Feeney, D. J. Mortlock, and N. Dalmasso, Clarifying the Hubble constant tension with a Bayesian hierarchical model of the local distance ladder, Mon. Not. R. Astron. Soc. 476, 3861 (2018); H.-Y. Wu and D. Huterer, Sample variance in the local measurements of the Hubble constant, Mon. Not. R. Astron. Soc. 471, 4946 (2017); S. Dhawan, S. W. Jha, and B. Leibundgut, Measuring the Hubble constant with Type Ia supernovae as near-infrared standard candles, Astron. Astrophys. 609, A72 (2018); C. A. P. Bengaly, U. Andrade, and J.S. Alcaniz, How does an incomplete sky coverage affect the Hubble constant variance?, Eur. Phys. J. C 79, 768 (2019); M. Rigault et al., Strong dependence of Type Ia supernova standardization on the local specific star formation rate, Astron. Astrophys. 644, A176 (2020); D. O. Jones, A. G. Riess, D. M. Scolnic, Y. C. Pan, E. Johnson, D. A. Coulter, K. G. Dettman, M. M. Foley, R. J. Foley, M. E. Huber, S. W. Jha, C. D. Kilpatrick, R. P. Kirshner, A. Rest, A. S. B. Schultz, and M. R. Siebert, Should Type Ia supernova distances be corrected for their local environments?, Astrophys. J. 867, 108 (2018); A. G. Riess, W. Yuan, S. Casertano, L. M. Macri, and D. Scolnic, The accuracy of the Hubble constant measurement verified through cepheid amplitudes, Astrophys. J. Lett. 896, L43 (2020); G. Efstathiou, A lockdown perspective on the Hubble tension (with comments from the SHOES team), arXiv:2007.10716.

[6] D. N. Spergel, R. Flauger, and R. Hložek, Planck data reconsidered, Phys. Rev. D 91, 023518 (2015); G. E. Addison, Y. Huang, D. J. Watts, C. L. Bennett, M. Halpern, G. Hinshaw, and J. L. Weiland, Quantifying discordance in the 2015 Planck CMB spectrum, Astrophys. J. 818, 132 (2016); G. Obied, C. Dvorkin, C. Heinrich, W. Hu, and V. Miranda, Inflationary features and shifts in cosmological parameters from Planck 2015 data, Phys. Rev. D 96, 083526 (2017); E. Calabrese, R. A. Hložek, J. R. Bond, M. J.
Devlin, J. Dunkley, M. Halpern, A. D. Hincks, K. D. Irwin, A. Kosowsky, K. Moodley, L. B. Newburgh, M. D. Niemack, L. A. Page, B. D. Sherwin, J. L. Sievers, D. N. Spergel, S. T. Staggs, and E. J. Wollack, Cosmological parameters from pre-Planck CMB measurements: A 2017 update, Phys. Rev. D 95, 063525 (2017); G. Efstathiou and S. Gratton, A detailed description of the CamSpec likelihood pipeline and a reanalysis of the Planck high frequency maps, arXiv:1910.00483; P. Motloch and W. Hu, Lensing-like tensions in the Planck legacy release, Phys. Rev. D 101, 083515 (2020); S. Aiola et al., The atacama cosmology telescope: DR4 maps and cosmological parameters, J. Cosmol. Astropart. Phys. 12 (2020) 047.

[7] G. E. Addison, D. J. Watts, C. L. Bennett, M. Halpern, G. Hinshaw, and J. L. Weiland, Elucidating $\Lambda$ CDM: Impact of baryon acoustic oscillation measurements on the Hubble constant discrepancy, Astrophys. J. 853, 119 (2018); DES Collaboration, Dark energy survey year 1 results: A precise $H_{0}$ measurement from DES Y1, BAO, and D/H Data, Mon. Not. R. Astron. Soc. 480, 3879 (2018); O. H. E. Philcox, M. M. Ivanov, M. Simonović, and M. Zaldarriaga, Combining full-shape and BAO analyses of galaxy power spectra: a 1.6\% CMB-independent constraint on $\mathrm{H}_{0}$, J. Cosmol. Astropart. Phys. 05 (2020) 032.

[8] W. Yuan, A. G. Riess, L. M. Macri, S. Casertano, and D. M. Scolnic, Consistent calibration of the tip of the red giant branch in the large magellanic cloud on the Hubble space telescope photometric system and a redetermination of the Hubble constant, Astrophys. J. 886, 61 (2019); C. D. Huang, A. G. Riess, W. Yuan, L. M. Macri, N. L. Zakamska, S. Casertano, P. A. Whitelock, S. L. Hoffmann, A. V. Filippenko, and D. Scolnic, Hubble space telescope observations of Mira variables in the SN Ia host NGC 1559: An alternative candle to measure the Hubble constant, Astrophys. J. 889, 5 (2020); K. C. Wong et al., H0LiCOW-XIII. A 2.4 per cent measurement of $\mathrm{H}_{0}$ from lensed quasars: $5.3 \sigma$ tension between early- and lateUniverse p, Mon. Not. R. Astron. Soc. 498, 1420 (2020); M. Millon et al., TDCOSMO. I. An exploration of systematic uncertainties in the inference of $\mathrm{H}_{0}$ from time-delay cosmography, Astron. Astrophys. 639, A101 (2020); D. W. Pesce, J. A. Braatz, M. J. Reid, A. G. Riess, D. Scolnic, J. J. Condon, F. Gao, C. Henkel, C. M. V. Impellizzeri, C. Y. Kuo, and K. Y. Lo, The Megamaser cosmology project. XIII. Combined Hubble constant constraints, Astrophys. J. Lett. 891, L1 (2020).

[9] W. L. Freedman, B. F. Madore, D. Hatt, T. J. Hoyt, I. S. Jang, R. L. Beaton, C. R. Burns, M. G. Lee, A. J. Monson, J. R. Neeley, M. M. Phillips, J. A. Rich, and M. Seibert, The Carnegie-Chicago Hubble program. VIII. An independent determination of the Hubble constant based on the tip of the red giant branch, Astrophys. J. 882, 34 (2019); W. L. Freedman, B. F. Madore, T. Hoyt, I. S. Jang, R. Beaton, M. G. Lee, A. Monson, J. Neeley, and J. Rich, Calibration of the tip of the red giant branch, Astrophys. J. 891, 57 (2020); S. Birrer, A. J. Shajib et al., TDCOSMO IV: Hierarchical time-delay cosmography-Joint inference of the Hubble constant and galaxy density profiles, Astron. Astrophys. 643, A165 (2020); S. S. Boruah, M. J. Hudson, and G. Lavaux, Peculiar velocities in the local Universe: 
Comparison of different models and the implications for $H_{0}$ and dark matter, arXiv:2010.01119.

[10] B. F. Schutz, Determining the Hubble constant from gravitational wave observations, Nature (London) 323, 310 (1986).

[11] D. E. Holz and S. A. Hughes, Using gravitational-wave standard sirens, Astrophys. J. 629, 15 (2005).

[12] N. Dalal, D. E. Holz, S. A. Hughes, and B. Jain, Short GRB and binary black hole standard sirens as a probe of dark energy, Phys. Rev. D 74, 063006 (2006).

[13] S. Nissanke, D. E. Holz, S. A. Hughes, N. Dalal, and J. L. Sievers, Exploring short gamma-ray bursts as gravitationalwave standard sirens, Astrophys. J. 725, 496 (2010).

[14] S. R. Taylor, J. R. Gair, and I. Mandel, Cosmology using advanced gravitational-wave detectors alone, Phys. Rev. D 85, 023535 (2012).

[15] C. Messenger and J. Read, Measuring a Cosmological Distance-Redshift Relationship Using Only Gravitational Wave Observations of Binary Neutron Star Coalescences, Phys. Rev. Lett. 108, 091101 (2012).

[16] S. Nissanke, D. E. Holz, N. Dalal, S. A. Hughes, J. L. Sievers, and C. M. Hirata, Determining the Hubble constant from gravitational wave observations of merging compact binaries, arXiv:1307.2638.

[17] M. Oguri, Measuring the distance-redshift relation with the cross-correlation of gravitational wave standard sirens and galaxies, Phys. Rev. D 93, 083511 (2016).

[18] W. Del Pozzo, T. G. F. Li, and C. Messenger, Cosmological inference using only gravitational wave observations of binary neutron stars, Phys. Rev. D 95, 043502 (2017).

[19] B. P. Abbott et al., A gravitational-wave standard siren measurement of the Hubble constant, Nature (London) 551, 85 (2017).

[20] N. Seto and K. Kyutoku, Prospects of the local Hubble parameter measurement using gravitational waves from double neutron stars, Mon. Not. R. Astron. Soc. 475, 4133 (2018).

[21] H.-Y. Chen, M. Fishbach, and D. Holz, A 2 per cent Hubble constant measurement from standard sirens within 5 years, Nature (London) 562, 545 (2018).

[22] M. Fishbach, R. Gray, I. Magaña Hernandez, H. Qi, A. Sur, F. Acernese, L. Aiello, A. Allocca, M. A. Aloy, and A. Amato, A standard siren measurement of the Hubble constant from GW170817 without the electromagnetic counterpart, Astrophys. J. Lett. 871, L13 (2019).

[23] S. M. Feeney, H. V. Peiris, A. R. Williamson, S. M. Nissanke, D. J. Mortlock, J. Alsing, and D. Scolnic, Prospects for Resolving the Hubble Constant Tension with Standard Sirens, Phys. Rev. Lett. 122, 061105 (2019).

[24] D. J. Mortlock, S. M. Feeney, H. V. Peiris, A. R. Williamson, and S. M. Nissanke, Unbiased Hubble constant estimation from binary neutron star mergers, Phys. Rev. D 100, 103523 (2019).

[25] R. Gray, I. Magaña Hernandez, H. Qi, A. Sur, P. R. Brady, H.-Y. Chen, W. M. Farr, M. Fishbach, J. R. Gair, A. Ghosh, D. E. Holz, S. Mastrogiovanni, C. Messenger, D. A. Steer, and J. Veitch, Cosmological inference using gravitational wave standard sirens: A mock data challenge, Phys. Rev. D 101, 122001 (2020).
[26] M. Soares-Santos et al., First measurement of the Hubble constant from a dark standard siren using the dark energy survey galaxies and the LIGO/Virgo binary-black-hole merger GW170814, Astrophys. J. Lett. 876, L7 (2019).

[27] A. Palmese et al., A statistical standard siren measurement of the Hubble constant from the LIGO/Virgo gravitational wave compact object merger GW190814 and dark energy survey galaxies, Astrophys. J. Lett. 900, L33 (2020).

[28] S. Mukherjee and B. D. Wandelt, Beyond the classical distance-redshift test: Cross-correlating redshift-free standard candles and sirens with redshift surveys, arXiv:1808 .06615 .

[29] S. Mukherjee, B. D. Wandelt, S. M. Nissanke, and A. Silvestri, Accurate and precision cosmology with redshift unknown gravitational wave sources, Phys. Rev. D 103, 043520 (2021).

[30] S. S. Vasylyev and A. V. Filippenko, A measurement of the Hubble constant using gravitational waves from the binary merger GW190814, Astrophys. J. 902, 149 (2020).

[31] H.-Y. Chen, C.-J. Haster, S. Vitale, W. M. Farr, and M. Isi, A standard siren cosmological measurement from the potential GW190521 electromagnetic counterpart ZTF19abanrhr, arXiv:2009.14057.

[32] V. Gayathri, J. Healy, J. Lange, B. O’Brien, M. Szczepanczyk, I. Bartos, M. Campanelli, S. Klimenko, C. Lousto, and R. O'Shaughnessy, Hubble constant measurement with GW190521 as an eccentric black hole merger, arXiv:2009 .14247.

[33] S. Mukherjee, A. Ghosh, M. J. Graham, C. Karathanasis, M. M. Kasliwal, I. Magaña Hernandez, S. M. Nissanke, A. Silvestri, and B.D. Wandelt, First measurement of the Hubble parameter from bright binary black hole GW190521, arXiv:2009.14199.

[34] S. Vitale and H.-Y. Chen, Measuring the Hubble Constant with Neutron Star Black Hole Mergers, Phys. Rev. Lett. 121, 021303 (2018).

[35] T. Dietrich, S. Khan, R. Dudi, S. J. Kapadia, P. Kumar, A. Nagar, F. Ohme, F. Pannarale, A. Samajdar, S. Bernuzzi, G. Carullo, W. Del Pozzo, M. Haney, C. Markakis, M. Pürrer, G. Riemenschneider, Y. E. Setyawati, K. W. Tsang, and C. Van Den Broeck, Matter imprints in waveform models for neutron star binaries: Tidal and self-spin effects, Phys. Rev. D 99, 024029 (2019).

[36] A. Matas, T. Dietrich, A. Buonanno, T. Hinderer, M. Pürrer, F. Foucart, M. Boyle, M. D. Duez, L. E. Kidder, H. P. Pfeiffer, and M. A. Scheel, Aligned-spin neutronstar-black-hole waveform model based on the effectiveone-body approach and numerical-relativity simulations, Phys. Rev. D 102, 043023 (2020).

[37] F. Foucart, T. Hinderer, and S. Nissanke, Remnant baryon mass in neutron star-black hole mergers: Predictions for binary neutron star mimickers and rapidly spinning black holes, Phys. Rev. D 98, 081501(R) (2018).

[38] B. P. Abbott et al., Prospects for observing and localizing gravitational-wave transients with Advanced LIGO, Advanced Virgo and KAGRA, Living Rev. Relativity 21, 3 (2018).

[39] LIGO, Virgo, and KAGRA Collaborations, https://dcc.ligo .org/ligo-t2000012/public. 
[40] LIGO Scientific and Virgo Collaborations et al., GWTC-1: A Gravitational-Wave Transient Catalog of Compact Binary Mergers Observed by LIGO and Virgo during the First and Second Observing Runs, Phys. Rev. X 9, 031040 (2019).

[41] See Supplemental Material at http://link.aps.org/ supplemental/10.1103/PhysRevLett.126.171102 for further detail on the waveform approximants, cosmographic relations and posterior employed in this work.

[42] M. U. Kruckow, T. M. Tauris, N. Langer, M. Kramer, and R. G. Izzard, Progenitors of gravitational wave mergers: Binary evolution with the stellar grid-based code COMBINE, Mon. Not. R. Astron. Soc. 481, 1908 (2018).

[43] R. Abbott et al., GWTC-2: Compact binary coalescences observed by LIGO and Virgo during the first half of the third observing run, arXiv:2010.14527.

[44] S. Typel, G. Röpke, T. Klähn, D. Blaschke, and H. H. Wolter, Composition and thermodynamics of nuclear matter with light clusters, Phys. Rev. C 81, 015803 (2010); M. Hempel and J. Schaffner-Bielich, A statistical model for a complete supernova equation of state, Nucl. Phys. A837, 210 (2010); T. Fischer, M. Hempel, I. Sagert, Y. Suwa, and J. Schaffner-Bielich, Symmetry energy impact in simulations of core-collapse supernovae, Eur. Phys. J. A 50, 46 (2014).

[45] F. Foucart, Black-hole-neutron-star mergers: Disk mass predictions, Phys. Rev. D 86, 124007 (2012).

[46] T. Binnington and E. Poisson, Relativistic theory of tidal Love numbers, Phys. Rev. D 80, 084018 (2009); P. Pani, L. Gualtieri, A. Maselli, and V. Ferrari, Tidal deformations of a spinning compact object, Phys. Rev. D 92, 024010 (2015); V. Cardoso, E. Franzin, A. Maselli, P. Pani, and G. Raposo, Testing strong-field gravity with tidal Love numbers, Phys. Rev. D 95, 084014 (2017); H. S. Chia, Tidal deformation and dissipation of rotating black holes, arXiv: 2010.07300 .

[47] P. Ajith, S. Babak, Y. Chen, M. Hewitson, B. Krishnan, J. T. Whelan, B. Brügmann, P. Diener, J. Gonzalez, M. Hannam, S. Husa, M. Koppitz, D. Pollney, L. Rezzolla, L. Santamaría, A. M. Sintes, U. Sperhake, and J. Thornburg, A phenomenological template family for black-hole coalescence waveforms, Classical Quantum Gravity 24, S689 (2007); P. Ajith, S. Babak, Y. Chen, M. Hewitson, B. Krishnan, A. M. Sintes, J. T. Whelan, B. Brügmann, P. Diener, N. Dorband, J. Gonzalez, M. Hannam, S. Husa, D. Pollney, L. Rezzolla, L. Santamaría, U. Sperhake, and J. Thornburg, Template bank for gravitational waveforms from coalescing binary black holes: Nonspinning binaries, Phys. Rev. D 77, 104017 (2008); M. Hannam, P. Schmidt, A. Bohé, L. Haegel, S. Husa, F. Ohme, G. Pratten, and M. Pürrer, Simple Model of Complete Precessing Black-HoleBinary Gravitational Waveforms, Phys. Rev. Lett. 113, 151101 (2014).

[48] A. Buonanno and T. Damour, Effective one-body approach to general relativistic two-body dynamics, Phys. Rev. D 59, 084006 (1999); Transition from inspiral to plunge in binary black hole coalescences, Phys. Rev. D 62, 064015 (2000); A. Bohé, L. Shao, A. Taracchini, A. Buonanno, S. Babak, I. W. Harry, I. Hinder, S. Ossokine, M. Pürrer, V. Raymond, T. Chu, H. Fong, P. Kumar, H. P. Pfeiffer, M. Boyle, D. A. Hemberger, L.E. Kidder, G. Lovelace, M. A. Scheel, and B. Szilágyi, Improved effective-one-body model of spinning, nonprecessing binary black holes for the era of gravitational-wave astrophysics with advanced detectors, Phys. Rev. D 95, 044028 (2017); E. Barausse and A. Buonanno, Improved effective-one-body Hamiltonian for spinning black-hole binaries, Phys. Rev. D 81, 084024 (2010).

[49] J.E. Thompson, E. Fauchon-Jones, S. Khan, E. Nitoglia, F. Pannarale, T. Dietrich, and M. Hannam, Modeling the gravitational wave signature of neutron star black hole coalescences, Phys. Rev. D 101, 124059 (2020).

[50] C. M. F. Mingarelli, J. Levin, and T. J. W. Lazio, Fast radio bursts and radio transients from black hole batteries, Astrophys. J. Lett. 814, L20 (2015).

[51] K. K. Y. Ng, S. Vitale, A. Zimmerman, K. Chatziioannou, D. Gerosa, and C.-J. Haster, Gravitational-wave astrophysics with effective-spin measurements: Asymmetries and selection biases, Phys. Rev. D 98, 083007 (2018).

[52] H.-Y. Chen, Systematic Uncertainty of Standard Sirens from the Viewing Angle of Binary Neutron Star Inspirals, Phys. Rev. Lett. 125, 201301 (2020).

[53] S. Rosswog, U. Feindt, O. Korobkin, M. R. Wu, J. Sollerman, A. Goobar, and G. Martinez-Pinedo, Detectability of compact binary merger macronovae, Classical Quantum Gravity 34, 104001 (2017).

[54] D. Scolnic et al. (DES Collaboration), How Many Kilonovae Can Be Found in Past, Present, and Future Survey Data Sets?, Astrophys. J. Lett. 852, L3 (2018).

[55] P. S. Cowperthwaite, V. A. Villar, D. M. Scolnic, and E. Berger, LSST target-of-opportunity observations of gravitational-wave events: Essential and efficient, Astrophys. J. 874, 88 (2019).

[56] C. N. Setzer, R. Biswas, H. V. Peiris, S. Rosswog, O. Korobkin, R. T. Wollaeger (LSST Dark Energy Science Collaboration), Serendipitous discoveries of kilonovae in the LSST main survey: Maximizing detections of subthreshold gravitational wave events, Mon. Not. R. Astron. Soc. 485, 4260 (2019).

[57] I. Mandel, W. M. Farr, and J. R. Gair, Extracting distribution parameters from multiple uncertain observations with selection biases, Mon. Not. R. Astron. Soc. 486, 1086 (2019).

[58] S. Vitale, D. Gerosa, W. M. Farr, and S. R. Taylor, Inferring the properties of a population of compact binaries in presence of selection effects, arXiv:2007.05579.

[59] W. J. Handley, M. P. Hobson, and A. N. Lasenby, polychord: Nested sampling for cosmology, Mon. Not. R. Astron. Soc. 450, L61 (2015); POLYCHORD: Next-generation nested sampling, Mon. Not. R. Astron. Soc. 453, 4384 (2015).

[60] G. Ashton et al., BILBY: A user-friendly Bayesian inference library for gravitational-wave astronomy, Astrophys. J. Suppl. Ser. 241, 27 (2019).

[61] Stan Development Team, PyStan: The python interface to stan, Version 2.19.1.1 (2019).

[62] M. Visser, Jerk, snap and the cosmological equation of state, Classical Quantum Gravity 21, 2603 (2004).

[63] J. Schreiber, Pomegranate: Fast and flexible probabilistic modeling in python, arXiv:1711.00137.

[64] H. Akaike, A new look at the statistical model identification, IEEE Trans. Autom. Control 19, 716 (1974). 
[65] T. A. Apostolatos, C. Cutler, G. J. Sussman, and K. S. Thorne, Spin-induced orbital precession and its modulation of the gravitational waveforms from merging binaries, Phys. Rev. D 49, 6274 (1994).

[66] C. Cutler and É.E. Flanagan, Gravitational waves from merging compact binaries: How accurately can one extract the binary's parameters from the inspiral waveform? Phys. Rev. D 49, 2658 (1994).

[67] A. Vecchio, LISA observations of rapidly spinning massive black hole binary systems, Phys. Rev. D 70, 042001 (2004).

[68] W. M. Farr, M. Fishbach, J. Ye, and D. E. Holz, A future percent-level measurement of the Hubble expansion at redshift 0.8 with Advanced LIGO, Astrophys. J. Lett. 883, L42 (2019).
[69] LIGO Scientific and the Virgo Collaborations et al., Binary black hole population properties inferred from the first and second observing runs of Advanced LIGO and Advanced Virgo, Astrophys. J. Lett. 882, L24 (2019).

[70] LIGO Scientific and the Virgo Collaborations, Population properties of compact objects from the second LIGO-Virgo gravitational-wave transient catalog, arXiv:2010.14533.

[71] Y. Huang, C.-J. Haster, S. Vitale, V. Varma, F. Foucart, and S. Biscoveanu, Statistical and systematic uncertainties in extracting the source properties of neutron star-black hole binaries with gravitational waves, arXiv:2005.11850.

[72] https://github.com/sfeeney/nsbh. 